\title{
Parallel perceptual channels at "deep" processing levels
}

\author{
GERALD T. GARDNER and DOUGLAS J. JOSEPH \\ University of Michigan, Dearborn, Michigan 48128
}

\begin{abstract}
This work extended the tachistoscopic paradigm developed by Shiffrin and Gardner (1972) to experimental tasks necessitating "deep" levels of perceptual processing. The results paralleled the Shiffrin and Gardner findings and supported the view that all perceptual analysis prior to decisional and memorial processing involves independent parallel channels.
\end{abstract}

Shiffrin and Gardner (1972) developed a new tachistoscopic detection paradigm-an extension of work by Eriksen and Spencer (1969)-in which the individual letters of a stimulus array are presented briefly at different time intervals, preceded and followed by a masking field. In one condition, four letters (one "target" and three "noise" letters) are exposed simultaneously for X msec; in a second condition, two of the letters are exposed for $\mathrm{X} \mathrm{msec}$, and the remaining two are exposed for $X$ msec following a 500-msec "intermission." The results obtained with this paradigm indicate equal target detection accuracy in the two conditions. If perceptual processing involved an attentional capacity limit (as postulated by Estes \& Taylor, 1966; and Rumelhart, 1970), performance should have been better in the sequential condition, as the subject could focus attention on only two of the letters at a time. The results thus suggest that withinfixation perceptual processing involves no attentional "bottleneck" or voluntary attentional control; instead, perception appears to occur in independent, parallel information channels.

Despite the methodological advantages of the above paradigm (see Gardner, 1973), its application has been limited so far to experiments incorporating relatively simple perceptual tasks: the detection of easy-todiscriminate target letters and/or detection performed at low levels of accuracy. This limitation raises an important question: Would parallel processing break down in paradigms requiring complete stimulus recognition, for example, whole-report tasks and other tasks necessitating "deeper" levels of perceptual analysis? Shiffrin and Gardner (1972) suggest that such a breakdown would not occur. Some reaction time data collected by Conner (1972), Egeth et al. $(1972,1973)$, and Estes (1972), however, have been interpreted as contradicting the Shiffrin and Gardner prediction.

This work was supported by USPHS Grant 1R03-MH2500401 and by Rackham Research Grant 360622 from the University of Michigan, Ann Arbor, awarded to the first author. The authors thank Howard Egeth, Emily Kirstein, and Richard Shiffrin for helpful comments on earlier drafts of this paper. The paper is sponsored by $W$. J. Thomson, who takes full editorial responsibility for its contents.
In the Connor experiments, subjects indicated which of two array types occurred on each trial: a "same" array containing $n$ identical letters vs. a "different" array containing one discrepant letter. In the Egeth et al. and Estes work, subjects attempted to detect a target character or characters appearing in an n-character stimulus array. The results obtained all followed the same general pattern. In conditions involving simple discriminations (i.e., when the discrepant or target characters were easily discriminable from the background characters), both response time and error rate remained invariant with $\mathrm{n}$ (Connor, 1972, Experiment I; Egeth et al., 1972, Experiment IV and pages 691-692 on Brand work; Estes, 1972, "NC" condition). These results parallel the Shiffrin and Gardner data in offering evidence for the operation of independent, perceptual channels. However, the invariance broke down in conditions involving discriminations that were difficult and performed with high accuracy; when discrepant or target characters were confusable with background characters, response times and/or error rates increased significantly with n (Connor, 1972, Experiment III; Egeth et al., 1973, Experiment I; Estes, 1972, "C" condition).

The theoretical implications of the above data are ambiguous. Connor (1972) interprets the results as supporting a two-stage perceptual model, such as that of Neisser (1967). In this model, crude analysis occurs at spatially parallel "pre-attentive" levels, whereas finer perceptual discriminations involve subsequent "focal attention" characterized by a spatial capacity limit. The "pre-attentive" mechanisms are sufficient for high accuracy on simple discriminations (and, presumably, low accuracy on difficult discriminations as in the Shiffrin \& Gardner, 1972, "high-confusability" condition), but "focal attention" must be called into play when both difficult discriminations and high accuracy are required.

Shiffrin and Gardner (1972), on the other hand, suggest that a theoretical position of the above type is ruled out by their data (see pages 81-82). In addition, it appears that the Connor-Egeth-Estes data are consonant with models in which all perceptual analysis occurs 
Table 1

Proportion of Correct Detections in Experiment I

\begin{tabular}{lccrcc} 
& \multicolumn{2}{c}{ Days 1 and 2} & & \multicolumn{2}{c}{ Days 3 and 4 } \\
\cline { 2 - 3 } \cline { 5 - 6 } Subject & Sim & Seq & & Sim & Seq \\
\hline P.C. & .938 & .930 & .859 & .867 \\
C.B. & .914 & .937 & .953 & .945 \\
B.N. & .913 & .921 & .906 & .906 \\
W.W. & .961 & .961 & .898 & .914 \\
Average & .932 & .937 & .904 & .908 \\
\hline
\end{tabular}

in independent, parallel channels. First, increases in reaction time and/or error rate with $\mathrm{n}$ are not inconsistent with an independent-parallel mechanism (see Egeth et al., 1972, 1973). Second, the increases with $\mathrm{n}$ could reflect the properties of decisional processes subsequent to independent-parallel perceptual analysis, as in the model developed by Shiffrin and Geisler (1973).

To resolve the ambiguity of the Connor-EgethEstes results, it seemed desirable to extend the Shiffrin and Gardner paradigm to tasks that would necessitate the mediation of "focal attention," should it exist. This extension was attempted in Experiments I and II below. As $\mathrm{n}$ remains constant in the Shiffrin and Gardner paradigm, the ambiguities inherent in the Connor, Egeth, and Estes data are bypassed; a model in which all perceptual analysis takes place in independent, parallel channels predicts equality of detection accuracy in simultaneous and sequential conditions, whereas a model involving any perceptual capacity limit predicts superior performance in the sequential condition (see Shiffrin \& Gardner, 1972; Shiffrin \& Geisler, 1973).

The involvement of "deep" perceptual levels was insured by requiring difficult perceptual discriminations, plus the highest performance accuracy consonant with the avoidance of ceiling effects. Experiment I was identical to the Shiffrin and Gardner (1972) Experiment III, high-confusability condition, except that exposure durations were adjusted to yield approximately $90 \%$ detection accuracy. Experiment II represented the Shiffrin and Gardner analog of Egeth et al. (1973) Experiment I and permitted a direct comparison with the ambiguous reaction time data discussed above.

\section{EXPERIMENT I}

Method

Subjects. Four female college-age volunteers were paid $\$ 2$ for each session.

Stimuli and Equipment. Stimuli consisted of arrays of four Para-Tipe characters, each character appearing at one corner of an imaginary square. One of the characters of each array was the "target" and was either "T" or " $F$;" the remaining characters were all T-F "hybrids." Individual characters were .3-deg in height, and adjacent characters were separated by $2.4 \mathrm{deg}$. Arrays were exposed at $.2 \mathrm{fL}$ luminance.

In the simultaneous condition, all four characters were exposed at the same time for a predetermined duration, preceded and followed by a masking-fixation array. This array contained a centered fixation dot and four square masking patterns, one pattern at each of the corner positions; each masking pattern subtended $.5 \mathrm{deg}$ and consisted of random pieces of the stimulus letters, similar to the "pattern mask" used by Turvey (1973). In the sequential condition, a single diagonal of the array (e.g., the upper left and lower right characters) was exposed for the same duration used in the simultaneous condition. Following a 500-msec delay, the other diagonal was exposed for the same duration as the first diagonal. Before the first diagonal, after the second, and during the 500 -msec-delay period, the masking-fixation array was exposed continuously.

Two decks of 32 stimulus arrays each were prepared, one for the simultaneous and one for the sequential condition.

Procedure. Each subject served in two exposure, three practice, and four experimental sessions. During the exposure sessions, a stimulus duration was determined so that the subject's detection accuracy was approximately $88 \%$. This duration was used through the practice and first two experimental sessions (average $=140 \mathrm{msec}$ ); based on performance in these sessions, a new duration was selected for the last two experimental sessions (average $=105 \mathrm{msec}$ ).

Each practice and experimental session consisted of one run through a 16-card practice deck, followed by two runs through each of the two stimulus decks. The order of arrays within a deck was randomized before each use. The order of presentation of simultaneous and sequential conditions was assigned on the basis of a Latin square design. At the beginning of each sequential deck, the subject was told which diagonal would be appearing first in the stimulus sequence.

On every trial the subject waited for the experimenter's ready signal, fixated the dot, and initiated the exposure by means of a hand switch. The subject then reported which target letter she thought was present in the array.

\section{EXPERIMENT II}

\section{Method}

Subjects. The four subjects from Experiment I served in Experiment II.

Stimuli and Equipment. Half of the stimulus arrays contained one target letter, an "S," and three randomly chosen non-S consonants; the remaining arrays contained four randomly chosen non-S consonants. Other aspects of stimuli and equipment were the same as in Experiment I, except that luminance was $.5 \mathrm{fL}$ and adjacent stimulus letters were separated by $1.8 \mathrm{deg}$.

Procedure. Each subject served in two exposure, one practice, and four experimental sessions. During the exposure sessions, a stimulus duration was determined so that the subject's detection accuracy approximated a $2.3 \mathrm{~d}_{\mathrm{s}}$ value (see below). This duration was used throughout the rest of the experiment (average $=60.9 \mathrm{msec}$ ).

On every trial the subject reported whether or not the target was present and indicated her degree of confidence with a 1-3 rating. Other procedural details were the same as in Experiment I.

\section{RESULTS}

The data from Experiment $\mathrm{I}$ are shown in Table 1. The entries indicate two-alternative forced-choice performance accuracy. Over all sessions, performance averaged .918 in the simultaneous condition and .923 in the sequential condition. An analysis of variance on arc-sine transformations of the data indicated that the difference between the two conditions was not significant. 
The data from Experiment II are summarized in Table 2 (table entries are in $d_{s}$ values, see Eriksen \& Spencer, 1969). Average performance accuracy was somewhat higher in the simultaneous condition than in the sequential condition. A t test indicated that this difference was not significant.

\section{DISCUSSION}

The outcomes of Experiments I and II may be summarized as follows: Subjects detected target letters with equal accuracy in the simultaneous and sequential conditions, in experimental paradigms requiring the performance of difficult perceptual discriminations at high levels of accuracy. The difficulty of discriminations required appears to have approximated that involved in the Connor-Egeth-Estes work. The task and stimulus vocabulary of Experiment II closely paralleled the Estes' "confusable" condition and the condition that yielded the greatest reaction time slope in the Egeth et al. work (1973, Experiment I). The target and "hybrid" noise characters of Experiment I were highly confusable (see Gardner, 1973), perhaps even more confusable than the letters used by Connor (Experiment III). Note that detection accuracy averaged over $90 \%$ in Experiment I, considerably higher than the analogous condition of Shiffrin and Gardner (1972). Performance averaged approximately $2.4 \mathrm{~d}_{s}$ in Experiment II, which corresponds to a .99 hit rate and a .10 false alarm rate; this is slightly better performance than in the analogous conditiop of Egeth et al. (1973, Experiment I), in which reaction time and error rate increased with $\mathrm{n}$.

The results of Experiments I and II appear to have the following theoretical implications for the Connor-Egeth-Estes data. According to Neisser (1967), difficult perœptual discriminations performed with high accuracy exceed the powers of "pre-attentive processes" and must be performed by "focal attention"-a "deeper" perceptual mechanism that has a spatial capacity limit. This, according to Connor (1972), is why in her experiments reaction time and error rate were invariant with $\mathrm{n}$ for simple discriminations, but increased with $\mathrm{n}$ for difficult discriminations. The Connor interpretation, however, seems contradicted by the fact that subjects in Experiments I and II performed difficult discriminations with equal (and high) accuracy when all stimulus letters had to be perceived simultaneously vs. when only two letters had to be perceived in the same interval; this equality should not be possible if the underlying perceptual mechanism involves a spatial capacity limit. The results of these experiments thus support the position of Shiffrin and Gardner (1972) with respect to "deep" levels of perceptual processing. It appears that perceptual analysis sufficient to accurately discriminate highly confusable alphanumeric stimuli occurs in independent, parallel channels; processing at this level seems to entail no spatial capacity limit ${ }^{\prime}$ or voluntary attentional control.
Table 2

Detection Accuracy in Experiment II (in $d_{s}$ Units)

\begin{tabular}{ccc}
\hline Subject & Sim & Seq \\
\hline P.C. & 2.09 & 1.93 \\
C.B. & 2.40 & 2.63 \\
B.N. & 3.67 & 2.47 \\
W.W. & 1.93 & 2.29 \\
Average & 2.52 & 2.33 \\
\hline
\end{tabular}

\section{REFERENCES}

Connor. J. Effects of increased processing load on parallel processing of visual displays. Perception \& Psychophysics, 1972, 12, 121-128.

Egeth, H., Jonides, J., \& Wall, S. Parallel processing of multi-element displays. Cognitive Psychology, 1972, 3. 674-698.

Egeth, H., Atkinson, J., Gilmore, G., \& Marcus, N. Factors affecting processing mode in visual search. Perception \& Psychophysics, 1973, 13, 394-402.

ERIKSEN, C., \& SPEnCer, T. Rate of information processing in visual perception. Journal of Experimental Psychology Monograph, 1969, 70(No. 2, Part 2).

EsTES, W. Interactions of signal and background variables in visual processing. Perception \& Psychophysics, 1972. 12. 278-286.

Estes, W., \& TAYlor, H. Visual detection in relation to display size and redundancy of critical elements. Perception \& Psychophysics, 1966, 1, 9-16.

GARDNER. G. Evidence for independent parallel channels in tachistoscopic perception. Cognitive Psychology, 1973, 4. 130-155.

NeIsser. U. Cognitive psychology. New York: AppletonCentury-Crofts. 1967

RUMelhart. D. A multicomponent theory of the perception of briefly exposed visual displays. Journal of Mathematical Psychology, 1970, 7, 191-218.

Shiffrin, R., \& GaRdNer, G. Visual processing capacity and attentional control. Journal of Experimental Psychology. 1972, 93, 72-83.

Shiffrin, R., \& Geisler, W. Visual recognition in a theory of information processing. In R. Solso (Ed.), The Loyola symposium: Contemporary vien'points in cognitive psychology. Washington, D.C: Winston, 1973.

TURVEY, $M$. On peripheral and central processes in vision: Inferences from an information-processing analysis of masking with patterned stimuli. Psychological Review, 1973, 80, 1-52.

\section{NOTE}

1. Excepting limits imposed by lateral masking or acuity (see Gardner, 1973).

(Received for publication August 5, 1975.) 